\title{
Ligand binding mode determination in Fragment Based Drug Discovery using X-ray crystallographic data coupled with QM/MM based refinement: Further applications of XModeScore
}

\author{
Oleg Y. Borbulevych ${ }^{\mathrm{a}}$ and Lance M. Westerhoff ${ }^{\mathrm{a}}$ \\ ${ }^{a}$ QuantumBio Inc., 2790 W. College Ave., State College, PA 16801, USA
}

Successful fragment based drug discovery/design (FBDD) is dependent upon accurate protein:ligand structure determination and characterization. Unfortunately, given the relatively small size of fragment molecules, coupled with the typically modest crystallographic resolutions routinely observed in FBDD campaigns and the weak or incomplete density around fragment molecules, accurate and unambiguous fragment placement within the relevant "blob" of electron density is often extremely difficult to achieve. We have developed a novel scoring method, called XModeScore, which incorporates both a rigorous statistical analysis of electron difference density distribution and the ligand strain energy. This method has been applied to correctly determine tautomeric/protonation states of ligands and active site residues, and in the present work, we have shown that it is also applicable to fragment placement. This method takes into account both residual X-ray difference density features around the ligand and the ligand strain energy to determine the mode which best fits the density out of a set of possible (e.g. docked) binding modes. We validated this approach against a diverse set of 55 pharmaceutically relevant protein-ligand (fragment) structures downloaded from PDB, and the high sensitivity of the XModeScore method was able to consistently find the correct fragment binding mode leading to heavyatom RMSDs as small as 0.02-0.05 $\AA$. 